\title{
Superior vena cava syndrome after creation of arteriovenous fistula
}

\author{
Engin Akgul, Sinan Erkul, Gulen Sezer Alptekin Erkul, Ahmet Hakan Vural \\ Department of Cardiovascular Surgery, Dumlupinar University, Evliya Celebi Training and Research Hospital, Kutahya, Turkey
}

\begin{abstract}
Patients with end-stage renal disease require vascular access for hemodialysis. Repeated insertions and longterm duration of permanent central venous catheters may cause venous stenosis and thrombosis in central veins, which may result in superior vena cava syndrome. A 38-year-old male patient with end-stage renal disease was admitted with complaint of dyspnea, edema of the face, neck and bilateral upper extremities. We had created a radiocephalic arteriovenous fistula at the level of left wrist two weeks ago, he had a permanent catheter in the right internal jugular vein for 2 months. The Doppler ultrasound and venographic examinations of central veins showed that the permanent catheter in the right internal jugular vein had caused the edema of the neck, face and bilateral upper extremities by narrowing the superior vena cava. The catheter was removed and the patient was anticoagulated. The patient's symptoms of shortness of breath were alleviated on hospital discharge and the swelling of the upper extremities diminished after one week. The hemodialysis patients should be directed to arteriovenous fistula surgery instead of central venous catheterization as soon as possible to avoid superior vena cava syndrome due to central venous catheters.
\end{abstract}

Eur Res J 2017;3(3):281-284

Keywords: Superior vena cava syndrome, central venous catheter, central venous stenosis, arteriovenous fistula, end-stage renal disease

Introduction

The obstruction of superior vena cava by thrombosis or external compression may cause superior vena cava syndrome (SVCS) [1]. The most common reason of external compression is intrathoracic neoplasm [2] whereas implantable central venous access devices and permanent catheters are the most common reasons of venous thrombosis $[1,2]$. When the obstruction begins within a chronical process, the collateral veins improve and the obstruction symptoms may be absent. However, the acute obstruction does not allow compensatory changes and results into SVCS which is defined as critical congestion that leads to edema on the face, neck, upper extremities and even to cerebral edema and herniation [2]. Vascular access for hemodialysis with a temporary or a permanent catheter is a well- 
known etiological risk factor for thrombosis. During insertion of the catheter, vascular endothelium is traumatized and it causes inflammation and coagulation finally it may cause intravascular thrombosis [3].

Arteriovenous fistula (AVF), as described by William Hunter in 1757 is an anomalous communication of the artery and the vein. An AVF may be related to trauma or surgical procedures also it may be congenital whereas it is created for vascular access in hemodialysis patients on purpose [4, 5]. However proximal vessel dilatation, pseudoaneurysm formation, rupture of fistula, cardiac overload and cardiac failure are some of the complications of AVFs [4].

\section{Case Presentation}

A 38-year-old male hemodialysis patient was hospitalized for creation of an AVF for long-term vascular access. He had a permanent catheter in the right internal jugular vein and a history of repeated central venous catheterization of bilateral subclavian and internal jugular veins. The size of the cephalic vein and the radial artery were sufficient at the level of wrist of the left upper extremity in the ultrasonographic imaging. A radiocephalic AVF was created with an end-to-side anastomosis technique with 7/0 polypropylene suture under local anesthesia. There was thrill on the location of created AVF. Two weeks after the operation the patient presented with progressive dyspnea and edema on the face, neck and bilateral upper extremities (Figure 1).

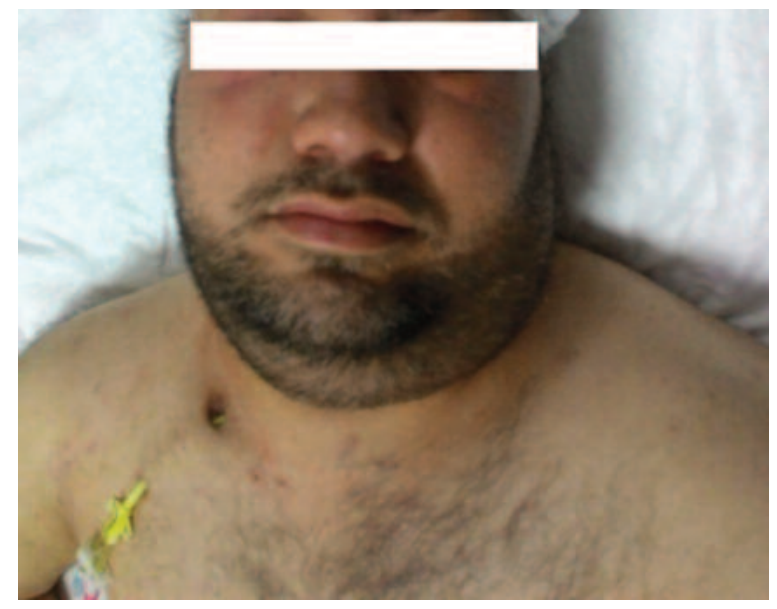

Figure 1. The edema at the face and neck. It can be seen especially at around the eyes.
The upper extremity veins were dilated bilaterally. He had tachypnea, tachycardia and crepitate rales at both lungs on admission. Doppler ultrasound and venography was performed. According to the doppler ultrasound findings, AVF and the related cephalic vein was patent and $272 \mathrm{ml}$ per minute flow measured. However venography showed that there was a narrowing starting from right internal jugular vein and continuing to the superior vena cava, due to the permanent catheter at this side (Figure 2).

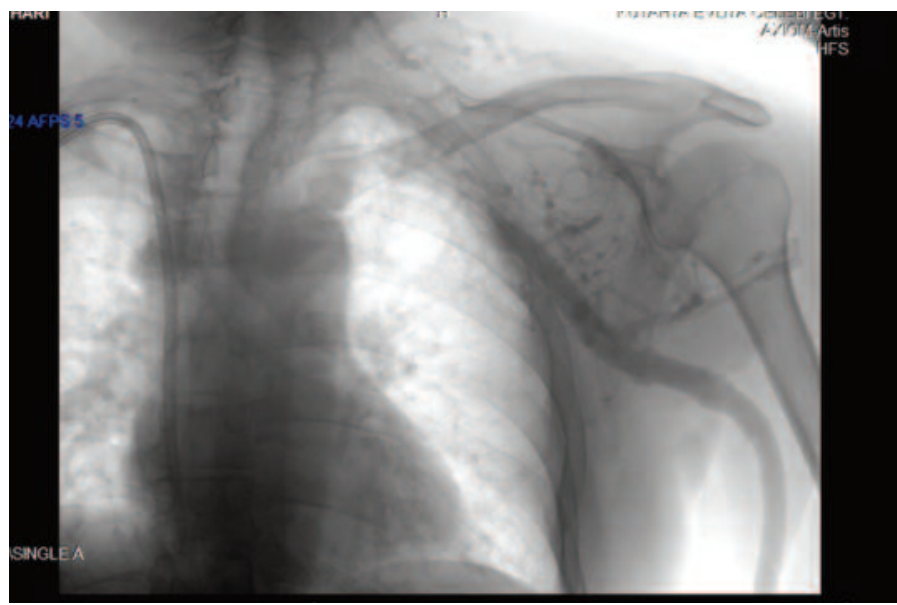

Figure 2. Permanent hemodialysis catheter, narrowed superior vena cava and presence of collateral veins.

Also mild stenosis could be seen at left side and collateral veins possibly due to the previous catheter insertions (Figure 3).

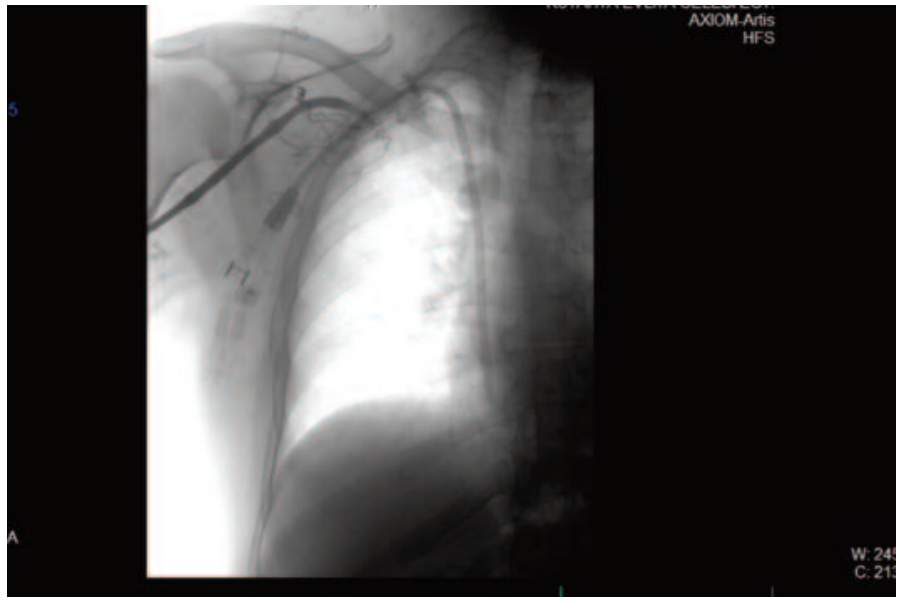

Figure 3. Venogram shows collateral veins with severe stenosis of the subclavian vein.

The flow were more than enough and the vessels had stenosis. These both conditions together caused the complaints of the patient. The permanent catheter in the right internal juguler vein was removed and right femoral vein catheterized with temporary catheter. Anti-aggregant and anticoagulant treatment 
was applied. Daily dosage of per oral $100 \mathrm{mg}$ acetylsalicylic acid (ASA) and 100 IU/kg subcutaneous enoxaparin sodium therapy was given. For long term anticoagulant therapy warfarin sodium was applied per oral according to the international normalized ratio (INR) adjusted level. After the removal of the catheter there has been a decrease in the swelling dramatically thus we decided to apply only medical treatment and avoid any further intervention. At discharge after three days he did not have any dyspneic symptoms more and after one week the edema on the face, neck and upper extremities was nearly totally diminished (Figure 4).

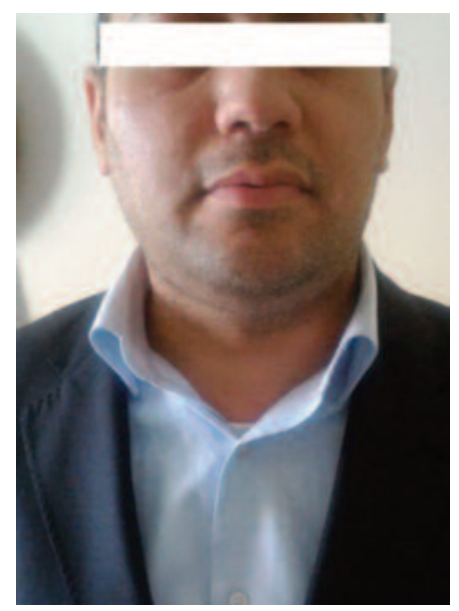

Figure 4. The edema diminished competely.

\section{Discussion}

Catheter-related SVCS is not a rare complication. It may develop because of hypercoagulability or stenosis / thrombosis in the superior vena cava. The reason is important because the treatment is shaped according to the reason. Removal of the catheter, anticoagulant, even thrombolytic treatment, percutaneous intervention could be the suitable treatment $[6,7]$. In our case patient relieved by removing the catheter so no any other procedure needed.

Central venous catheters are related to thrombosis, infections, bacteremia, SVCS and pneumonia [8]. There are many kinds of vascular access devices but the complications related to hemodialysis catheters (HD) are more often. Particularly permanent catheters have a long term duration and a great internal lumen width which may facilitate thrombosis and stenosis of the vessel. There are also patient related factors such as venipuncture trauma, vein caliber, thromboembolic event history, malignancy and hypercoagulative diseases [8].

According to the recommendations of American College of Chest Physicians for the treatment of catheter-associated thrombosis; catheter should be removed and the patient should be undergone anticoagulant treatment at least for three months [8]. But if the catheter is functional and cannot be removed anticoagulant treatment should be continued as long as catheter remains [8].

By the technological developments new treatment methods such as thrombus aspiration, mechanical thrombolysis, transluminal balloon angioplasty and venous stenting may be applied in appropriate patients [9]. Our patient's compliations were significally reduced after removing the catheter and the AV fistula was still working well. So percutaneous intervention not considered.

\section{Conclusion}

Patients with end-stage renal disease are at risk of SVCS and pulmonary thromboembolism due to catheter-related thrombosis. These are life-threatening complications. Also repeated vascular interventions may be harmful for central venous structures even if there is not intravascular clotting. We think that hemodialysis patients should be directed to AVF creation as soon as possible and the patients should be prepared for an AVF from the time of diagnosis for avoiding multiple catheter insertions. Particularly autogenous AVF is the most comfortable and safe way of hemodialysis vascular access.

\section{Informed consent}

Written informed consent was obtained from the patient for the publication of this case report.

\section{Conflict of interest}

The authors declared that there are no potential conflicts of interest with respect to the research, authorship, and/or publication of this article.

\section{References}

[1] Zaki M, Hynes N, Alawy M, Sultan S. The first case using synthetic vein for jugular to iliac vein bypass to treat superior vena cava obstruction: clinical dilemma and literature review JAVA 2015;20:92-6.

[2] Sriramnaveen P, Kumar VS, Kishore CK, Sai Naresh VV, 
Sivaramakrishna G, Manjusha Y, et al. Delayed presentation of superior vena cava syndrome after hemodialysis catheter removal. Saudi J Kidney Dis Transp 2011;22:554-6.

[3] Akoglu H, Yilmaz R, Peynircioglu B, Arici M, Kirkpantur A, Cil $\mathrm{B}$, et al. A rare complication of hemodialysis catheter: superior vena cava syndrome. Hemodial Int 2007;11:385-91.

[4] Williams AE, Sinha P. Arteriovenous fistula: a rare complication of peripheral venous cannulation and an example of the importance of clinical examination. Med Rep Case Stud 2016;1:105.

[5] Chang HH, Chang YK, Lu CW, Huang CT, Chien CT, Hung $\mathrm{KY}$, et al. Statins improve long term patency of arteriovenous fistula for hemodialysis. Sci Rep 2016;6:22197.

[6] Cui J, Kawai T, Irani Z. Catheter-directed thrombolysis in acute superior vena cava syndrome caused by central venous catheters. Semin Dial 2015,28:548-51.

[7] Siegel Y, Kuker R. Superior vena cava obstruction in hemodialysis patients: symptoms, clinical presentation and outcomes compared to other etiologies. Ther Apher Dial 2016;20:390-3.

[8] Thapa S, Terry PB, Kamdar BB. Hemodialysis catheterassociated superior vena cava syndrome and pulmonary embolism: a case report and review of the literature. BMC Res Notes 2016;9:233.

[9] Sista AK, Vedantham S, Kaufman JA, Madoff DC. Endovascular interventions for acute and chronic lower extremity deep venous disease: state of the art. Radiology 2015;276:31-53. 